\title{
On the Two-Equal-Disjoint Path Cover Problem of Crossed Cubes
}

\author{
Pao-Lien Lai ${ }^{\natural *}$, Hong-Chun $\mathrm{Hsu}^{\sharp}$
}

\author{
${ }^{\natural}$ Department of Computer Science and Information Engineering \\ National Dong Hwa University, Shoufeng, Hualien, Taiwan 97401, R.O.C. \\ $\sharp$ Department of Computer Science and Communication Engineering \\ Providence University, Shalu, Taiwan 43301 R.O.C.
}

\begin{abstract}
Embedding of paths have attracted much attention in the parallel processing. Many-to-many communication is one of the most central issues in various interconnection networks. A graph $G$ is globally two-equal-disjoint path coverable if for any two distinct pairs of vertices $(u, v)$ and $(w, x)$ of $G$, there exist two disjoint paths $P$ and $Q$ satisfied that (1) $P$ joins $u$ to $v$ and $Q$ joins $w$ to $x,(2)$ $|P|=|Q|$, and $(3) V(P \cup Q)=V(G)$. In this paper we prove that $C Q_{n}$ is globally 2-equal-disjoint path coverable for $n \geq 5$.
\end{abstract}

Keywords: Interconnection network; Crossed cube; disjoint path; $k$-equal-disjoint path cover, 2-equal-disjoint path coverable.

\section{Introduction}

For the graph definition and notation we follow [1]. $G=$ $(V, E)$ is a graph if $V$ is a finite set and $E$ is a subset of $\{(a, b) \mid(a, b)$ is an unordered pair of $V\}$. We say that $V$ is the vertex set and $E$ is the edge set. A path of length $k$ from $x$ to $y$ is a finite sequence of distinct vertices $\left\langle v_{0}, v_{1}, v_{2}, \ldots, v_{k}\right\rangle$, where $x=v_{0}, y=v_{k}$, and $\left(v_{i-1}, v_{i}\right) \in E$ for all $1 \leq i \leq k$. For convenience, we use the sequence $\left\langle v_{0}, \ldots, v_{i}, P, v_{j}, \ldots, v_{k}\right\rangle$, where $P=$ $\left\langle v_{i}, v_{i+1}, \ldots, v_{j}\right\rangle$ to denote the path $\left\langle v_{0}, v_{1}, v_{2}, \ldots, v_{k}\right\rangle$. Note that it is possible that the path $P$ has length 0 . We can also write the path $\left\langle v_{0}, v_{1}, v_{2}, \cdots, v_{k}\right\rangle$ as $\left\langle v_{0}, P_{1}, v_{i}, v_{i+1}, \cdots, v_{j}, P_{2}, v_{t}, \cdots, v_{k}\right\rangle$, where $P_{1}$ is the path $\left\langle v_{0}, v_{1}, \cdots, v_{i}\right\rangle$ and $P_{2}$ is the path $\left\langle v_{j}, v_{j+1}, \cdots, v_{t}\right\rangle$. We use $d(u, v)$ to denote the distance between $u$ and $v$, i.e., the length of the shortest path joining $u$ and $v$.

${ }^{*}$ Correspondence to: Assistant Professor Pao-Lien Lai, Department of Computer Science and Information Engineering, National Dong Hwa University, Shoufeng, Hualien, Taiwan 97401, R.O.C. email:baolein@mail.ndhu.edu.tw

This work was supported in part by the National Science Council of the Republic of China under Contract NSC 94-2213-E-259-026.
A path is a Hamiltonian path if it contains all vertices of $G$. A graph $G$ is Hamiltonian connected if there exists a Hamiltonian path joining any two distinct vertices. A cycle is a path (except the first vertex is the same as the last vertex) containing at least three vertices. A cycle of $G$ is a Hamiltonian cycle if it contains all vertices. A graph is Hamiltonian if it has a Hamiltonian cycle.

Finding node-disjoint paths is one of the important issues of routing among nodes in various interconnection networks. Node-disjoint (abbreviated as disjoint) paths can be used to avoid communication congestion and provide parallel paths for an efficient data routing among nodes. Moreover, multiple disjoint paths can be more fault-tolerant of node or link failures and greatly enhance the transmission reliability. Disjoint paths generally fall into three categories: one-to-one, one-to-many, and manyto-many. The one-to-one disjoint path is built with one source and one destination. The one-to-many disjoint paths like a tree structure, they contain one source and many distinct destination nodes. The many-to-many disjoint paths involve $k, k \geq 1$, disjoint paths with $k$ pairs distinct source and destination nodes.

A disjoint path cover in a graph $G$ is to find disjoint paths containing all the vertices in $G$. For an embedding of linear arrays in a network, the cover implies every node can be participated in a pipeline computation. One-toone disjoint path covers in recursive circulants [10] and one-to-many disjoint path covers in some hypercube-like interconnection networks [11] were studied. The manyto-many $k$-disjoint path cover is proposed by Park etc. in [12]. In this paper, we call such many-to-many $k$ disjoint path cover (abbreviated as $k$-disjoint path cover) as many-to-many $k$-equal-disjoint path cover (abbreviated as $k$-equal-disjoint path cover) that $k$ disjoint paths have same lengths. The $k$ disjoint paths with equal length implies that the parallel processing of $k$ pipeline is guaranteed accurately. Furthermore, a graph is called globally $k$-equal-disjoint path coverable if there exists a $k$-equaldisjoint path cover for any $k$ distinct source-destination pairs. 
An n-dimensional crossed cube, $C Q_{n}$ [3], is a variation of hypercube, which is derived from hypercube by changing the connection of some hypercube links. Though some topological properties of crossed cubes have been studied in the literature $[2,3,4,5,6,7,8,9,15,16]$. In this paper, we prove that the crossed cube is globally twoequal-disjoint path coverable. In next section, we give the definition of two-equal-disjoint path coverable problem and Crossed Cubes. Then we prove that the crossed cube is globally two-equal-disjoint path coverable in the section 3. In the final section, we give the conclusion.

\section{Preliminary}

In this section, we will first give the definition of globally two-equal-disjoint path coverable problem of a graph $G$, and then we will give the relevant definitions in graph theory and the definition of the Crossed cubes.

Definition 1 A graph $G$ is $(u, v, w, x)$-two-equal-disjoint path coverable if there are two disjoint paths $P$ and $Q$ such that $P$ joins the vertices $u$ to $v, Q$ joins the vertices $w$ to $x$, and $V(P \cup Q)=V(G)$.

Definition 2 A graph $G$ is globally two-equal-disjoint path coverable if for any two distinct pairs of vertices $(u, v)$ and $(w, x)$, the $(u, v, w, x)$-two-equal-disjoint path cover exist.

To define the Crossed cubes, as proposed by Efe [3], the notion so called "pair related" relation is introduced.

\section{Definition 3}

Let $R=\{(00,00),(10,10),(01,11),(11,01)\}$. Two twodigit binary strings $u=u_{1} u_{0}$ and $v=v_{1} v_{0}$ are pair related if and only if $(u, v) \in R$.

The following is the recursive definition of the $n$ dimensional Crossed cube $C Q_{n}$.

Definition 4 [3] The Crossed cube $C Q_{1}$ is a complete graph with two nodes labelled by 0 and 1 , respectively. For $n \geq 2$, an $n$-dimensional Crossed cube $C Q_{n}$ consists of two $(n-1)$-dimensional sub-Crossed cubes, $C Q_{n-1}^{0}$ and $C Q_{n-1}^{1}$, and a perfect matching between the nodes of $C Q_{n-1}^{0}$ and $C Q_{n-1}^{1}$ according to the following rule:

Let $V\left(C Q_{n-1}^{0}\right)=\left\{0 u_{n-2} u_{n-3} \ldots u_{0}: u_{i}=0\right.$ or 1$\}$ and $V\left(C Q_{n-1}^{1}\right)=\left\{1 v_{n-2} v_{n-3} \ldots v_{0}: v_{i}=0\right.$ or 1$\}$. The node $u=0 u_{n-2} u_{n-3} \ldots u_{0} \in V\left(C Q_{n-1}^{0}\right)$ and the node $v=1 v_{n-2} v_{n-3} \ldots v_{0} \in V\left(C Q_{n-1}^{1}\right)$ are adjacent in $C Q_{n}$ if and only if

(1) $u_{n-2}=v_{n-2}$ if $n$ is even, and

(2) $\left(u_{2 i+1} u_{2 i}, v_{2 i+1} v_{2 i}\right) \in R$, for $0 \leq i<\left\lfloor\frac{n-1}{2}\right\rfloor$.
If $u$ and $v$ are two adjacent vertices in $C Q_{n}$ and $j$ is the leftmost differing bit, we say that $v$ is the $j$-neighbor of $u$. Moreover, $\bar{u}$ represents the $(n-1)$-neighbor of $u$ in $C Q_{n}$. We then introduce a important fault Hamiltonian result for proving the main theorem in the next section of this paper. A graph $G$ is $k$-fault Hamiltonian connected if for any faulty set $F \subset V(G) \cup E(G)$ such that $|F| \leq k$, $G-F$ is still Hamiltonian connected.

Lemma 1 [7] $C Q_{n}$ is $n-3$ fault Hamiltonian connected.

\section{Crossed cube is globally two- disjoint equal path coverable}

As a starting point we present the lemma below which establishes the base case of Theorem 1 .

Lemma $2 C Q_{3}$ and $C Q_{4}$ are not globally two-equaldisjoint path coverable.

Proof. To prove this lemma, we give a counter example for each case. Given two pair of vertices 0,1 and 2,3, there is no two-equal-disjoint path and cover all vertices in $C Q_{3}$. Given two pair of vertices 0,3 and 4,7 , there is no two-equal-disjoint path and cover all vertices in $C Q_{4}$ also.

Lemma $3 C_{5}$ is globally two-equal-disjoint path coverable.

Proof. To prove this case is very tedious. With long and detail discussion, we have completed theoretical proof for $C Q_{5}$. Nevertheless, we do not present it in this paper for reducing complexity. However, we can also verify this small case directly using computer.

Next we formally show the main result that $C Q_{n}, n \geq$ 5 , is globally two-disjoint equal path coverable.

Theorem 1 Crossed cube, $C Q_{n}$, is globally two-equaldisjoint path coverable for $n \geq 5$.

Proof. We prove this theorem by induction on $n$. The base case is $C Q_{5}$. With Lemma 3, the base case holds. By induction hypothesis, we can assume that $C Q_{n}$ is globally two-equal-disjoint path coverable. Now, we need to show that $C Q_{n+1}$ is also globally two-equal-disjoint path coverable. Let $(a, b)$ and $(c, d)$ be two distinct sourcedestination pairs of $C Q_{n+1}$. In the following, we establish two disjoint paths $P, Q$ of length $2^{n}-1$ with end vertices $(a, b)$ and $(c, d)$, respectively. By the relative positions of the four vertices, we divide the proof into four cases as follows.

Case 1: $a, b, c$ and $d$ are all in same $C Q_{n}$, say $C Q_{n}^{0}$, of $C Q_{n+1}$. 
By induction, there are two disjoint paths $\left(a, P_{0}, b\right)$ and $\left(c, Q_{0}, d\right)$ in $C Q_{n}^{0}$, where $\left|P_{0}\right|=\left|Q_{0}\right|=2^{n-1}-$ 1. Let $(w, x)$ and $(y, z)$ be two edge on $P_{0}$ and $Q_{0}$, respectively, and let $P_{0}=\left(a, P_{0}^{1}, w, x, P_{0}^{2}, b\right)$ and $Q_{0}=\left(c, Q_{0}^{1}, y, z, Q_{0}^{2}, d\right)$. By induction again, we have two disjoint paths $P_{1}$ and $Q_{1}$ of length $2^{n-1}-1$ with end vertices $\bar{w}, \bar{x}, \bar{y}$ and $\bar{z}$ in $C Q_{n}^{1}$. Let $P=\left(a, P_{0}^{1}, w, \bar{w}, P_{1}, \bar{x}, x, P_{0}^{2}, b\right)$ and $Q=$ $\left(c, Q_{0}^{1}, y, \bar{y}, Q_{1}, \bar{z}, z, Q_{0}^{2}, d\right)$. Clearly, $P$ and $Q$ are two disjoint paths and $|P|=|Q|=2^{n}-1$. (See Fig. 1)

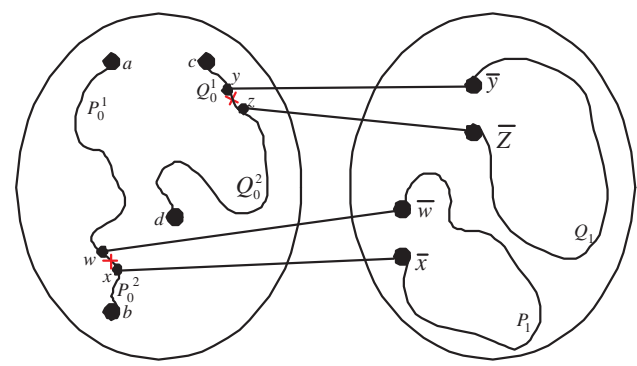

Figure 1: $a, b, c$ and $d$ are in $C Q_{n}^{0}$.

Case 2: $a, b$ and $c$ are in same $C Q_{n}$, say $C Q_{n}^{0}$, of $C Q_{n+1}$; $d$ is in $C Q_{n}^{1}$.

Let $x$ be a vertex in $C Q_{n}^{0}$ and $x \notin\{a, b, c, \bar{d}\}$. By hypothesis, there are two disjoint paths $\left\langle a, P_{0}, b\right\rangle$ and $<c, Q_{0}, x>$ with $\left|P_{0}\right|=\left|Q_{0}\right|=2^{n-1}-1$ in $C Q_{n}^{0}$. Let $w$ be the neighbor of $b$ on the path $P_{0}$. If $\bar{w} \neq d$ and $\bar{b} \neq d$, we can get two disjoint paths $\left\langle\bar{w}, P_{1}, \bar{b}>\right.$ and $<\bar{x}, Q_{1}, d>$ with $\left|P_{1}\right|=\left|Q_{1}\right|=2^{n-1}-1$ in $C Q_{n}^{1}$. (See Fig. 2) Let $P=<a, P_{0}, w, \bar{w}, P_{1}, \bar{b}, b>$ and $Q=<$ $c, Q_{0}, x, \bar{x}, Q_{1}, d>$. In this case, $P$ and $Q$ are also two disjoint paths with $|P|=|Q|=2^{n}-1$. However, if $\bar{w}=d$ or $\bar{b}=d$. Choosing $a$ and the neighbor of $a$ on the path $P_{0}$ to replace $b$ and $w$, we can rebuild another two-equal-disjoint path cover by the similar technique.

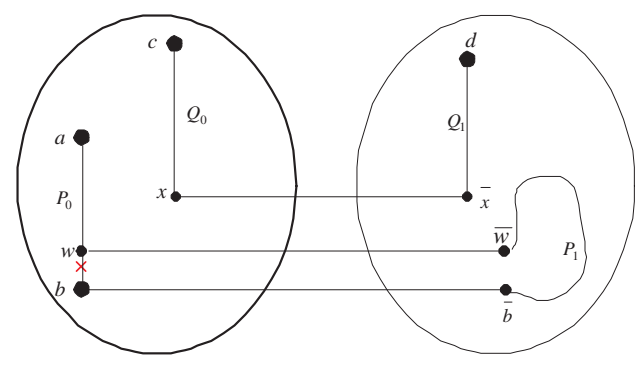

Figure 2: $a, b$ and $c$ are in $C Q_{n}^{0} ; d$ is in $C Q_{n}^{1}$.

Case 3: $a$ and $b$ are both in same $C Q_{n}$, say $C Q_{n}^{0}$, of $C Q_{n+1} ; c$ and $d$ are both in $C Q_{n}^{1}$.
By Lemma 1, there exists a Hamiltonian path $P(Q$ resp.) joining $a(c$ resp. $)$ and $b(d$ resp. $)$ in $C Q_{n}^{0}\left(C Q_{n}^{1}\right.$ resp.). (See Fig. 3)
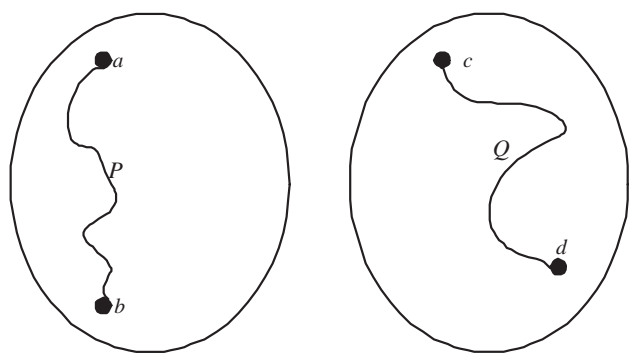

Figure 3: $a$ and $b$ are in $C Q_{n}^{0} ; c$ and $d$ are in $C Q_{n}^{1}$.

Case 4: $a$ and $c$ are both in same $C Q_{n}$, say $C Q_{n}^{0}$, of $C Q_{n+1} ; b$ and $d$ are both in $C Q_{n}^{1}$.

Let $w$ and $x$ be any two distinct vertices in $C Q_{n}^{0}$ except $a$ and $c$ and $\bar{w} \notin\{b, d\}, \bar{x} \notin\{b, d\}$. By hypothesis, there are two disjoint paths $\left\langle a, P_{0}, w\right\rangle$ and $\left\langle c, Q_{0}, x\right\rangle$ with $\left|P_{0}\right|=\left|Q_{0}\right|=2^{n-1}-1$ in $C Q_{n}^{0}$. Similarly, there are two disjoint paths $<\bar{w}, P_{1}, b>$ and $<\bar{x}, Q_{1}, d>$ with $\left|P_{1}\right|=$ $\left|Q_{1}\right|=2^{n-1}-1$ in $C Q_{n}^{1}$. Let $P=<a, P_{0}, w, \bar{w}, P_{1}, b>$ and $Q=<c, Q_{0}, x, \bar{x}, Q_{1}, d>$. In this case, $P$ and $Q$ are also two disjoint paths with $|P|=|Q|=2^{n}-1$. (See Fig. 4)

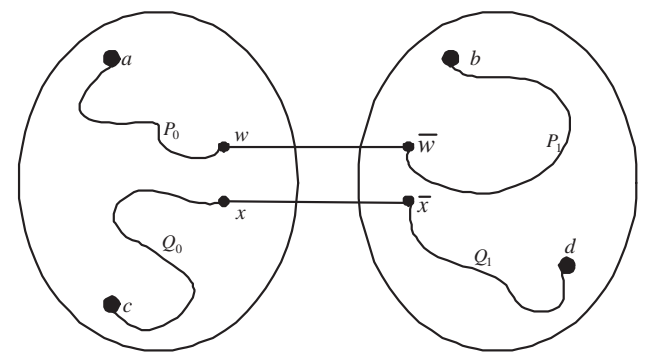

Figure 4: $a$ and $c$ are in $C Q_{n}^{0} ; b$ and $d$ are in $C Q_{n}^{1}$.

\section{Conclusion}

In this paper, we discussed the two-equal-disjoint path coverable problem and proved that Crossed Cubes $C Q_{n}$ are globally two-equal-disjoint path coverable for $n \geq 5$. The globally two-equal-disjoint path coverable problem is a extension of Hamiltonian connected problem. We can see Hamiltonian connected problem as globally one-path coverable problem, and then we extended this property 
to globally two-equal-disjoint path coverable. This work may help to discuss the many-to-many disjoint path coverable problem.

\section{References}

[1] J. A. Bondy and U. S. R. Murty, Graph Theory with Applications, North Holland, New York, 1980.

[2] C. P. Chang, T. Y. Sung, and L. H. Hsu,"Edge Congestion and Topological Properties of Crossed Cubes," IEEE Trans. Parallel and Distributed Systems, vol. 11, no. 1, pp. 64-80, 2000.

[3] K. Efe,"The Crossed Cube Architecture for Parallel Computing," IEEE Trans. Parallel and Distributed Systems, vol. 3, no. 5, pp. 513-524, 1992.

[4] K. Efe,"A Variation on the Hypercube with lower Diameter," IEEE Trans. Computers, vol. 40, no. 11, pp. 1,312-1,316, 1991.

[5] K. Efe, P. K. Blackwell, W. Slough, and T. Shiau, "Topological Properties of the Crossed Cube Architecture," Parallel Computing, vol. 20, pp. 1,763$1,775,1994$.

[6] J. Fan, X. Lin, and X. Jia, “Optimal Path Embedding in Crossed Cubes," IEEE Trans. Parallel and Distributed Systems, vol. 16, no. 12, pp. 1,190-1,200, 2005.

[7] W.T. Huang, Y.C. Chuang, L.H. Hsu, and J.M. Tan, "On the Fault-Tolerant Hamiltonicity of Crossed Cubes," IEICE Transaction on Fundamentals, Vol. E85-A, pp. 1359-1371, 2002.

[8] P. Kulasinghe, "Connectivity of the Crossed Cube," Information Processing Letters, vol. 61, pp. 221226, 1997.

[9] P. Kulasinghe and S. Bettayeb, "Embedding Binary Trees into Crossed Cubes," IEEE Trans. Computers, vol. 44, no. 7, pp. 923-929, 1995.

[10] J. H. Park, "One-to-one disjoint path covers in recursive circulants," Journal of KISS, 30(12), pp. 691698, 2003.

[11] J. H. Park, "One-to-many disjoint path covers in a graph with faulty elements," in Proc. of the International Computing and Combinatorics Conference COCOON 2004, pp. 392- 401, 2004.

[12] J. H. Park, "Many-to-many disjoint path covers in a graph with faulty elements," International Symposium on Algorithms and Computation, ISAAC 2004, pp. 742-753, 2004.
[13] C.-H. Tsai, "Linear array and ring embeddings in conditional faulty hypercubes," Theoretical Computer Science, 314, pp. 431-443, 2004.

[14] C. H. Tsai, J. J. M. Tan, and L. H. Hsu, "The superconnected property of recursive circulant graphs," Inform. Proc. Lett., 91(6), pp. 293-298, 2004.

[15] J. M. Xu, M. Ma, and M. Lü, "Paths in Möbius cubes and Crossed cubes," Information Processing Letters, vol. 16, no. 12, pp. 1,190-1,200, 2005.

[16] S. Q. Zheng and S. Latifi, "Optimal Simulation of Linear Multiprocessor Architectures on MultiplyTwisted Cube Using Generalized Gray Code," IEEE Trans. Parallel and Distributed Systems, vol. 7, no. 6, pp. 616-619, 1996. 